\title{
Medidas de porosidad en mortero de cemento y en hormigón
}

B. LICHTNER, Ingeniero y J. HUNDT, Conferenciante. Berlín

Tonindustrie Zeitung: n० 8, págs. 232.236

\section{INTRODUCCION}

La porosidad del hormigón, en la que, por una parte influye, el valor agua/cemento (valor a/c) dado previamente, y por otra, está sometida a variaciones según crece la edad del hormigón (1), es de importancia decisiva para el desarrollo de sus propiedades tecnológicas. Las modificaciones de la estructura de los poros pueden intervenir para definir el progreso de la madurez, que se caracteriza por la hidrólisis, hidratación y disposición.

Objeto de los análisis efectuados en el Instituto del Vidrio, Cerámica y Aglomerantes de la Universidad Técnica de Berlín y del Centro Federal para Comprobación de Materiales (BAM) de Berlín, fueron las mediciones de porosidad en el mortero de cemento y hormigón en función del valor a/c y la edad.

\section{PROCEDIMIENTO DE MEDIDA}

Schwiete y Ludwig (2) indican los siguientes procedimientos para las mediciones de porosidad:

1. Procedimiento de absorción de agua para poros de radios $>$ de $10^{3} \AA$, aproximadamente.

2. Procedimiento de medida de la porosidad por mercurio, para poros de radios comprendidos entre 75 y $75 \times 10^{3} \AA$, aproximadamente.

3. Procedimiento de circulabilidad para poros de radios $>$ de $10^{3} \AA$, aproximadamente.

4. Procedimiento de difusión para poros de radios $>$ de $10 \AA$, aproximadamente.

5. Procedimiento de sorción para poros de radios comprendidos entre 10 y $200 \AA$, aproximadamente.

En los tiempos más recientes Astbury y Vyse (3) han desarrollado otro método para las investigaciones de porosidad. En este procedimiento se determina en el material que se ensaya la velocidad de la absorción capilar en un electrólito acuoso diluido y después de conseguir la saturación se mide la permeabilidad bajo presión exterior. Según indicación de los autores, el procedimiento es adecuado para la captación de poros de radios $>$ de $500 \AA$, aproximadamente.

En el presente planteamiento se aplicaron 2 procedimientos de medida para determinación de las distribuciones de magnitudes de poros:

1. ${ }^{\circ} \quad$ El procedimiento del porosímetro de mercurio, según Ritter y Drake (4), para lo que se dispuso de un aparato de la firma Carlo Erba con una presión de trabajo máxima de 2.000 atmósferas. Esto corresponde a una gama de medidas para poros de radios comprendidas entre $37,5 \AA$ con 2.000 atmósferas y $75 \times 10^{3} \AA$ a una atmósfera. 


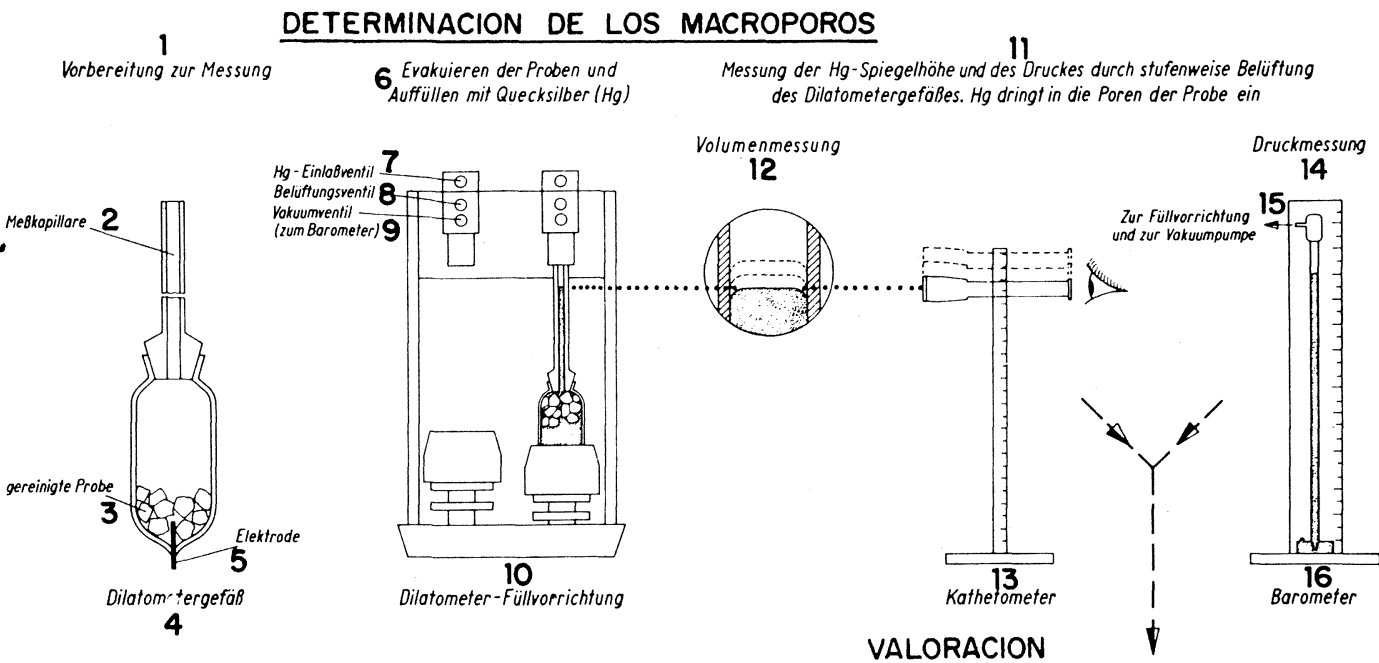

$\begin{aligned} r & =\frac{2 \sigma \cdot \cos \theta}{p} \\ v_{m} & =\frac{A\left(h_{t}-h_{p}\right)}{-\left(h_{t}-h_{p}\right) 100} \\ u_{r a, b} & =\frac{h_{t}}{n}\end{aligned}$

17

DETERMINACION DE LOS MICROPOROS Übernahme des DilatometergefäBes
von der Bestimmung der Makro-Poren $\quad 19 \begin{aligned} & \text { Messung der Volumina von Poren mit abnehmenden Äquivalentradien. } \\ & \text { Ourch Druckerhöhung dringt } \mathrm{Hg} \text { in die Poren der Probe ein }\end{aligned}$

20 Volumenmessung über $\mathrm{Hg}$-Niveau-Fühler Druckmessung

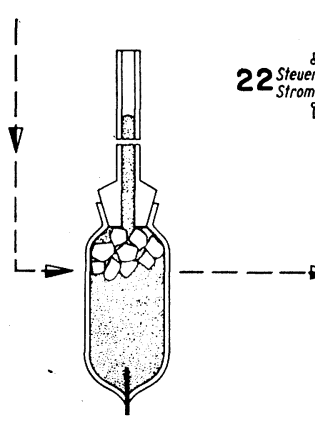

i.

$$
\begin{gathered}
\text { Dilatometergefäb. } \\
\text { gefüllt mit Probe und } \mathrm{Hg}
\end{gathered}
$$
18

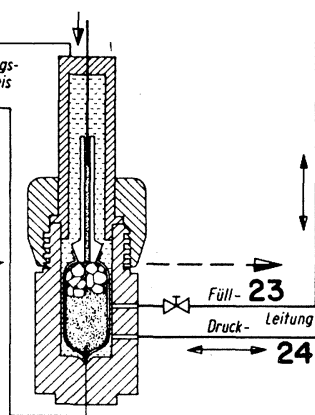

25
1. Preparación para la medida.

2. Capilar de medida.

3. Muestra purificada.

4. Vasija del dilatómetro.

5. Electrodo.

6. Evacuación de las probetas y llenado de mercurio.

7. Válvula de entrada del mercurio.

8. Válvula de salida del aire.

9. Válvula de vacío (para el barómetro)

10. Dispositivo de llenado del dilatómetro.

11. Medida de la altura del nivel de $\mathrm{Hg}$ y de la presión mediante evacuación gradual del recipiente del dilatómetro. El $\mathrm{Hg}$ penetra en los poros del material a ensayar.

12. Medida' del volumen

13. Catetómetro.

14. Medida de la presión.

15. Hacia el dispositivo de llenado y a la bomba de vacío.

16. Barómetro.

17. Recepción de la vasija del dilatómetro antes de la determinación de los macroporos.

18. Vasija del dilatómetro, llena con el material a ensayar y $\mathrm{Hg}$.

Registriereinrichtung

27. Bomba.

28. Alcohol

29. Aceite

36. Diagrama.

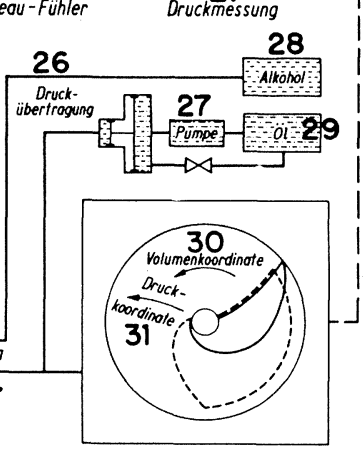

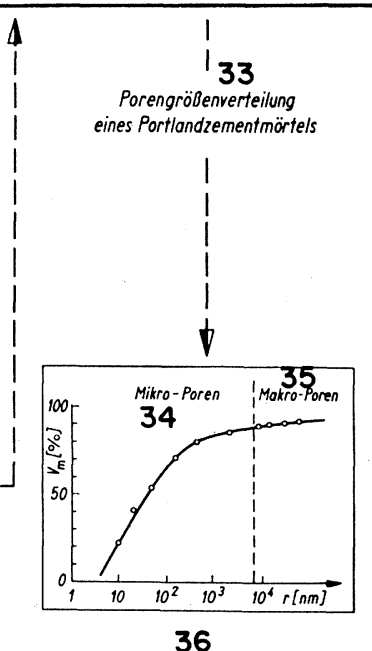

19. Medida de los volúmenes de los poros con radios equivalentes en disminución. Mediante el aumento de la presión penetra el $\mathrm{Hg}$ en los poros del material a ensayar.

20. Medida del volumen con la sonda de nivel de $\mathrm{Hg}$.

21. Medida de presión.

22. Circuito de corriente de mando.

23. Dirección de llenado.

24. Dirección de presión.

25. Recipiente de presión para la medida.

26. Transmisión de la presión.

30. Coordenada de volumen.

31. Coordenada de presión.

32. Dispositivo de registro

33. Distribución de la magnitud de los poros de un mortero de cemento portland.

34. Microporos.

35. Macroporos.

Fig. 1.-Esquema del porosímetro de mercurio para la determinación de la distribución de las magnitudes de poros (según K. Niesel, BAM). 
2. ${ }^{\circ}$ El procedimiento de sorción indicado por Cranston e Inkley (5) y modificado por Orr y Dalla Valle (6), en el que la distribución de los radios de poros se averigua mediante utilización de isotermas de sorción de $\mathrm{N}_{2}$.

El procedimiento del porosímetro de mercurio ha sido descrito por numerosos autores (2), (4), (7). El esquema del procedimiento así como el desarrollo de su utilización se halla descrito en la figura 1.

En el procedimiento de sorción hay que calcular la distribución del tamaño de los poros con ayuda de la ecuación de Kelvin partiendo de los volúmenes de gas absorbidos o desorbidos de un punto de medida a otro punto de medida. Para obtener el verdadero radio de los poros, se deben corregir los radios ya calculados teniendo en cuenta los espesores de capa absorbidos en las paredes de los poros. En el procedimiento que se utiliza se parte del hecho de que se trata de poros cilíndricos. En el curso de las investigaciones efectuadas se han medido los volúmenes absorbidos de $\mathrm{N}_{2}$, tanto por el procedimiento volumétrico como también por el gravimétrico.

En la figura 2 se ha representado el aparato que funciona según el procedimiento volumétrico. En los émbolos de absorción (A) se introduce el material que se ensaya hasta un máximo de $500 \AA$. Después de su evacuación se introduce por medio de la bureta graduada (B) nitrógeno dosificado en el émbolo de absorción, debiéndose medir la presión por medio del catetómetro en los meniscos de mercurio.
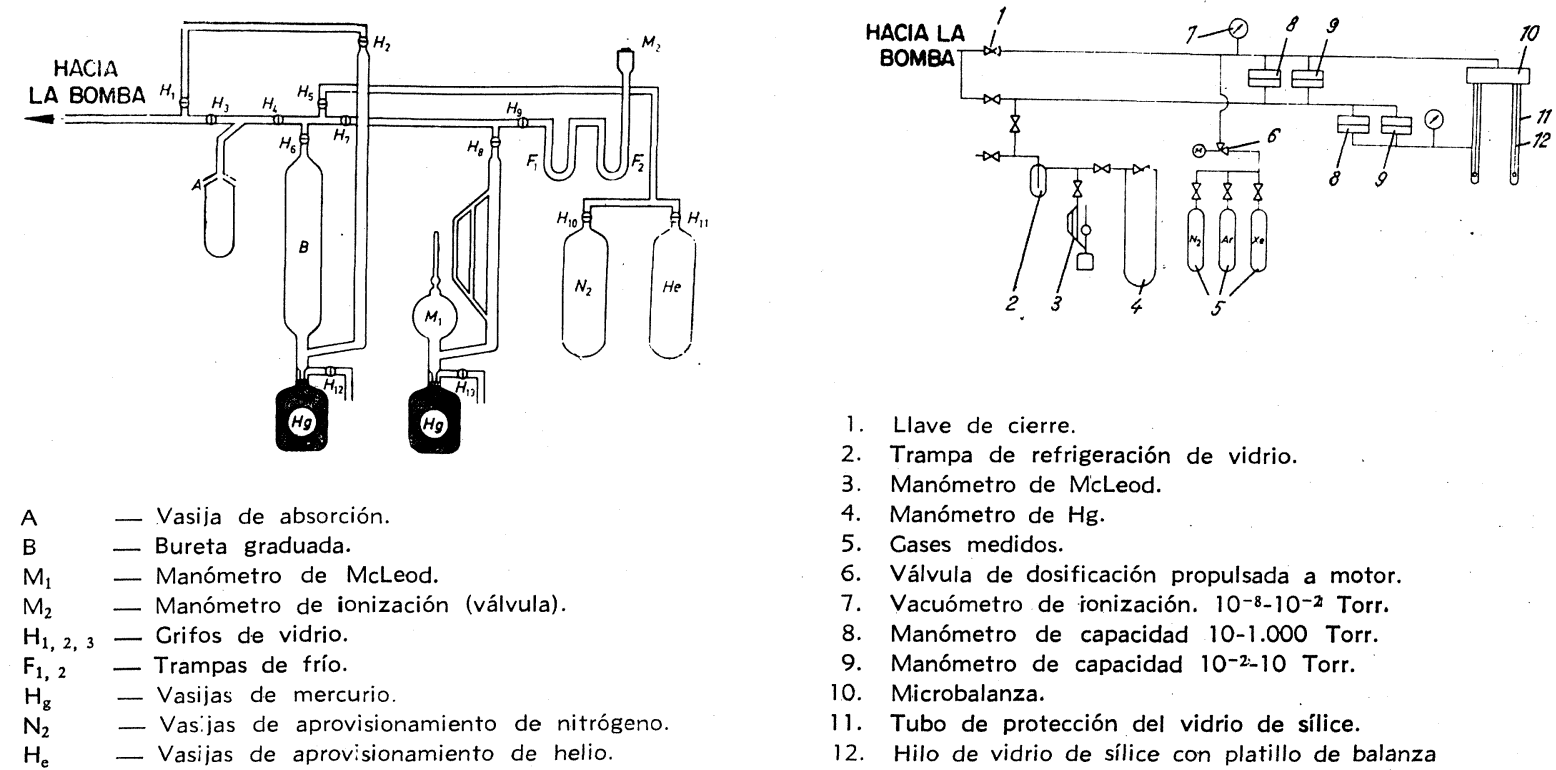

1. Llave de cierre.

2. Trampa de refrigeración de vidrio.

3. Manómetro de McLeod.

4. Manómetro de $\mathrm{Hg}$

5. Gases medidos.

6. Válvula de dosificación propulsada a motor.

7. Vacuómetro de ronización. $10^{-8-10^{-2}}$ Torr.

8. Manómetro de capacidad 10-1.000 Torr.

9. Manómetro de capacidad 10-2-10 Torr.

10. Microbalanza.

11. Tubo de protección del vidrio de sílice.

12. Hilo de vidrio de sílice con platillo de balanza

Fig. 2.-Esquema de la disposición de ensayo para la medición volumétrica de la absorción de $\mathbf{N}_{2}$.
Fig. 3.-Esquema de la disposición de ensayo para la medición gravimétrica de la absorción de $\mathrm{N}_{2}$.

La figura 3 muestra el aparato para la determinación gravimétrica de los volúmenes sorbidos de gas. La microbalanza eléctrónica permite el análisis de volúmenes con muestra de hasta aproximadamente 2 gramos, que dispone el manómetro de capacidad utilizado para la medición de presión sobre una gama de medida de 10 a 1.000 Torr. Después de la evacuación, el gas llega al sistema de medida a través de una válvula de dosificación propulsada a motor. 
En comparación con el método de medida volumétrica el método gravimétrico presenta la ventaja de que la presión y el volumen de gas sorbido se pueden medir independientemente entre sí.

\section{REALIZACION DE LOS ENSAYOS}

Para el ensayo se emplean tres morteros de cemento con valores a/c de 0,45, 0,50 y 0,55 y dos hormigones con valores a/c de 0,50 y 0,55 , para cuya preparación se emplearon P-450 y arena o gravilla como árido.

Según DIN 1164 se prepararon prismas con dimensiones de $4 \mathrm{~cm} \times 4 \mathrm{~cm} \times 16 \mathrm{~cm}$ y cubos con $20 \mathrm{~cm}$ de arista, se desmoldaron al cabo de un día y se almacenaron, para alcanzar el tiempo conveniente de ensayo, a $20^{\circ} \mathrm{C}$ y aproximadamente a $100 \%$ de humedad relativa del aire.

Para cumplir condiciones equivalentes de ensayos, se tuvo que extraer el agua absorbida por las superficies de los materiales que se ensayan antes de comenzar las investigaciones o análisis de porosidad. Los correspondientes ensayos previos dieron como resultado que con un secado al vacío efectuado durante 48 horas a $10^{6}$ Torr y a $20^{\circ} \mathrm{C}$ se pudieron crear condiciones de partida suficientemente equivalentes en el material a ensayar desmenuzado o machacado.

Los problemas que hay que aclarar durante la deshidratación del material que se ensaya antes de la investigación de la porosidad se hallan estrechamente relacionados a la cuestión de la diferencia del agua unida molecularmente y químicamente. Al igual que la estructura de los poros, también el contenido de agua unida químicamente es una medida del progreso de madurez de la pasta de cemento. Según Copeland y Hayes (8), el volumen del agua que permanece en la pasta de cemento con muy poca presión de vapor después del secado (predominantemente unida en forma química) es aproximadamente proporcional a la parte de cemento hidratado. El volumen del agua unida químicamente puede intervenir por consiguiente para caracterizar el grado de hidratación o el progreso de madurez. El procedimiento de secado indicado por los dos autores para determinar el agua molecular se basa en un peso a alto vacío y a una temperatura de $20^{\circ} \mathrm{C}$, habiéndose aplicado adicionalmente perclorato de magnesio como agente de secado.

Kroone y Blakey (9) llevaron a cabo ensayos de secado a $108^{\circ} \mathrm{C}$ y 760 Torr, que produjeron aproximadamente iguales resultados. Como no se puede excluir sin embargo en el hormigón fresco variaciones de estructura al aumentar la temperatura, no fue conveniente la aplicación de un sencillo procedimiento de secado. En la figura 4 se han agrupado algunos resultados de los ensayos de secado. Los ensayos se efectuaron a un vacío de $10^{-6}$ Torr en hormigones de diferente edad. Se indican las pérdidas de peso en función del tiempo de secado. De esta representación hay que concluir que la proporción de agua enlazada molecularmente y químicamente varía al aumentar la edad y que el progreso del secado del hormigón, de diferentes edades de envejecimiento, presenta diferente comportamiento al aumentar la temperatura.

Los conocimientos que se pueden obtener exclusivamente de los ensayos de secado permiten sólo una afirmación limitada sobre el progreso de madurez, pues con éstos no se pueden captar directamente las variaciones que se desarrollan en la pasta del cemento. Las tomas efectuadas con el microscopio electrónico de retícula permiten dirigir una mirada en la estructura de los poros que en especial interesa aquí. La figura 5 muestra pasta de cemento con poros, que se encuentran ya en la zona de captación de los procedimientos porosimétricos de mercurio. En la figura, que ha sido aumentada en un factor de 50 (fig. 6), se pueden reconocer hendiduras de un orden de magnitud, determinadas ya con ayuda de las mediciones de sorción. 


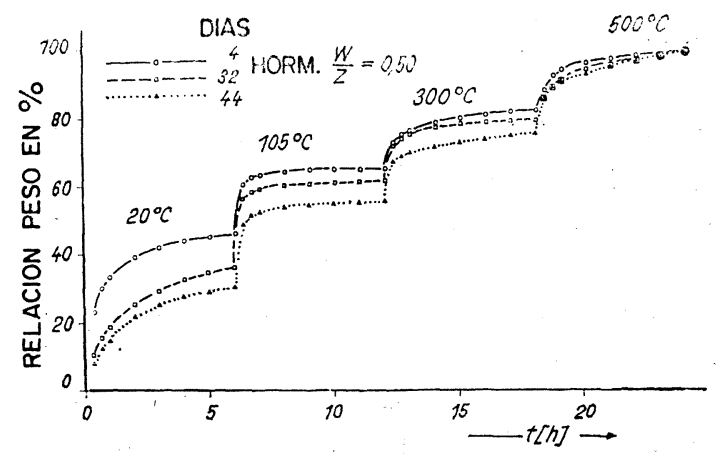

Fig. 4.-Pruebas de deshidratación con hormigón $(a / c=$ $=0,50$ ) de diferente edad en un aparato de vacío con equipo de micropesaje.
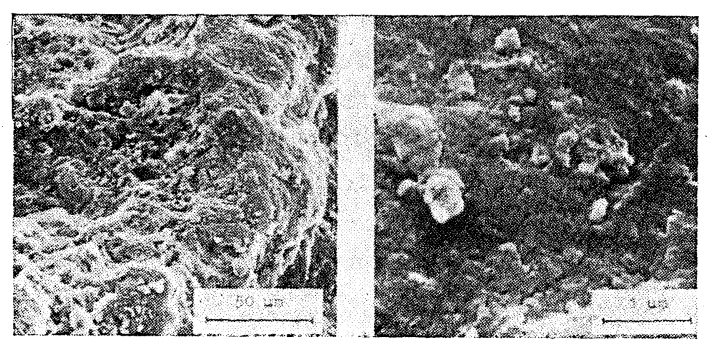

Fig. 5.-Tomas de la estructura porosa de la pasta de cemento con ayuda del microscopio electrónico de retícula.
Fig. 6.-Tomas de la estructura porosa de la pasta de cemento con ayuda del microscopio electrónico de retícula.

\section{RESULTADOS DE LA INVESTIGACION}

\subsection{Análisis con el procedimiento del porosímetro de mercurio}

La figura 7 presenta las magnitudes de poros medidas con el porosímetro de mercurio en el hormigón con un valor a/c de 0,50 con una edad de 4 y 28 días, representada en función de la porosidad total. En la figura 8 se han registrado los mismos valores de medida como distribuciones de magnitud de poros, pudiéndose conocer perfectamente que la parte de los pequeños radios de poros es mayor en el ensayo a 28 días de duración que en el ensayo a 4 días de duración. Esta tendencia se ha podido comprobar con todos los materiales de ensayo analizados. Además es característico que al aumentar la edad del hormigón la reducción de la pendiente de las curvas en la zona comprendida entre unos 100 y 500 \& resulta mayor. Los máximos de las distribuciones de magnitud de poros no pudieron comprobarse con este aparato - las líneas de finas rayas de la figura 8- indican los límites de captación del método de medida empleado. Respecto de los diferentes valores a/c analizados no resultan diferencias esenciales. Las variaciones debidas al diferente valor a/c se encuentran en el presente caso claramente dentro de los límites de error del método de medida.

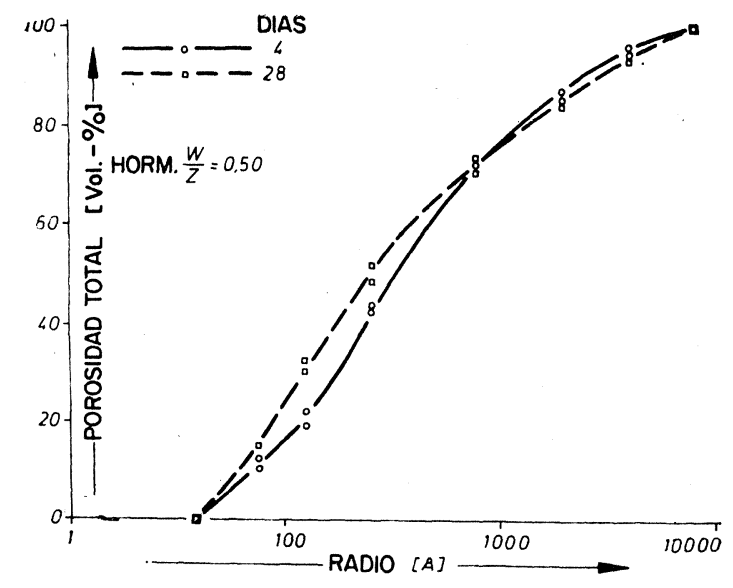

Fig. 7.-Procedimiento de porosímetro de mercurio; radios de poros en función de la porosidad total respecto del hormigón $(a / c=0,50)$ de edad de 4 y 28 días.

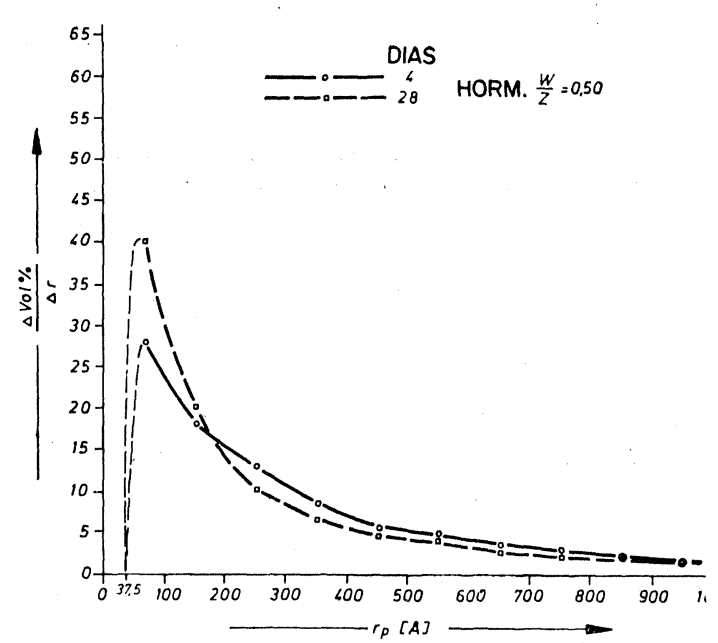

Fig. 8.-Procedimiento de porosímetro de mercurio; distribuciones de los radios de los poros en el hormigón $(a / c=0,50)$ de edad de 4 y 28 días. 


\subsection{Investigaciones con el procedimiento de sorción de $\mathbf{N}_{2}$}

La figura 9 muestra las isotermas de absorción de $\mathrm{N}_{2}$ medidas en el hormigón de valor a/c de 0,50 , respecto a diferentes etapas de edad por procedimiento volumétrico, isotermas, que fueron referidas a un volumen de la probeta de 100 gramos cada vez. Las distribuciones de magnitud de poros representadas en la figura 10 y averiguadas con estas mediciones permiten conocer claramente cómo se desplazan los máximos al aumentar la edad del hormigón hacia los poros de pequeño radio y, además, que la superficie dentro de la zona del radio de poro se va haciendo mayor en conjunto entre aproximadamente $10 \mathrm{y}$ $20 \AA$. En la figura 11 se han representado isotermas medidas mediante el procedimiento gravimétrico en los mismos materiales de ensayo, pero con edades de 4 y 36 días, y concretamente en cada caso, las ramas de absorción y las correspondientes ramas de desorción referidas a $100 \mathrm{mg}$ de contenido de aglomerante. Mientras que en el segundo caso de medida (material de prueba con 4 días de edad) se cierra la histéresis, esto no ocurre en el primer paso (material de ensayo de 36 días de edad). A este fenómeno se ha hecho ya referencia en la literatura (12). Hay que atribuir seguramente este fenómeno a variaciones estructurales de las superficies, que han sido provocadas por el nitrógeno absorbido. Los resultados reproducidos en la figura 14 respecto del hormigón con el valor a/c de 0,55 corresponden a los de la figura 11.

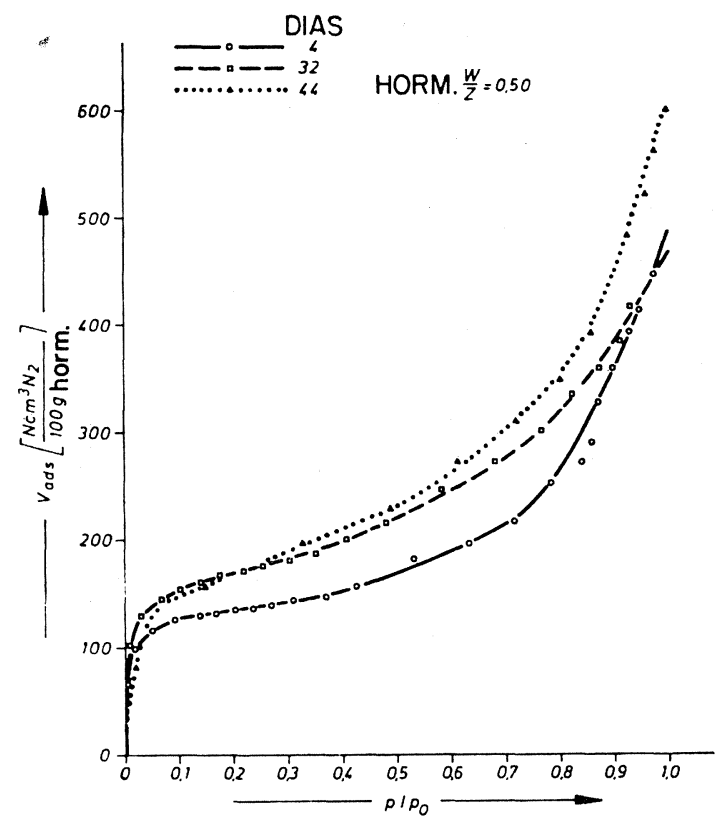

Fig. 9.-Evaluación volumétrica de la absorción de $\mathrm{N}_{2}$; isotermas de absorción de diferentes etapas de edad de un hormigón $(a / c=0,50)$.

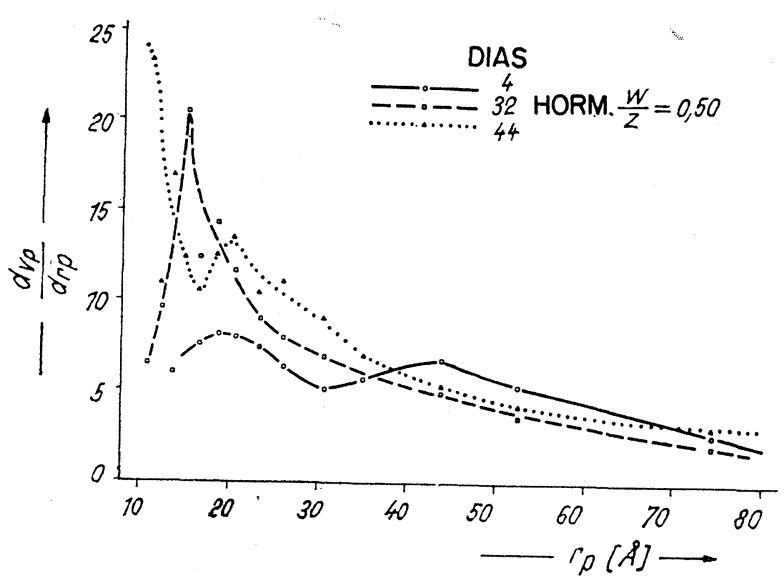

Fig. 10.-Evaluación volumétrica de la absorción de $\mathrm{N}_{2}$; distribución de los radios de poros en diferentes edades de un hormigón $(a / c=0,50)$, calculada a partir de las isotermas de absorción.

Las isotermas de absorción medidas con ayuda del procedimiento gravimétrico siguen el tipo $\mathrm{V}$ de la distribución propuesta para el procedimiento de BET (10), (11). Al igual que las medidas publicadas por Mikhail, Copeland y Brunauer (12) las isotermas de absorción registradas aquí mediante procedimiento volumétrico no presentan coincidencia alguna con el tipo IV. Según Lippens y de Boer (13), en la carga de una superficie plana no porosa resulta una dependencia entre el volumen absorbido y el espesor de la capa estática, que obedece a una función lineal, y partiendo de cuyo declive se puede calcular directamente la superficie específica. Según los dos autores, en el caso de superficies porosas pueden presentarse variaciones de la recta tanto hacia arriba ("condensación capilar") como hacia abajo ("llenado de los poros finos"). 
En la figura 13 se han comparado entre sí las diferentes distribuciones de la magnitud de poros, obtenidas en el hormigón con valores de a/c 0,50 y 0,55 , con ayuda del procedimiento gravimétrico. Las diferentes edades muestran las características ya descritas. En la probeta con valor de a/c 0,55 y edad de 33 días el máximo se encuentra por debajo del de la probeta correspondiente a 8 días de edad, pero con un radio de poro que es menor que aproximadamente $10 \AA$.

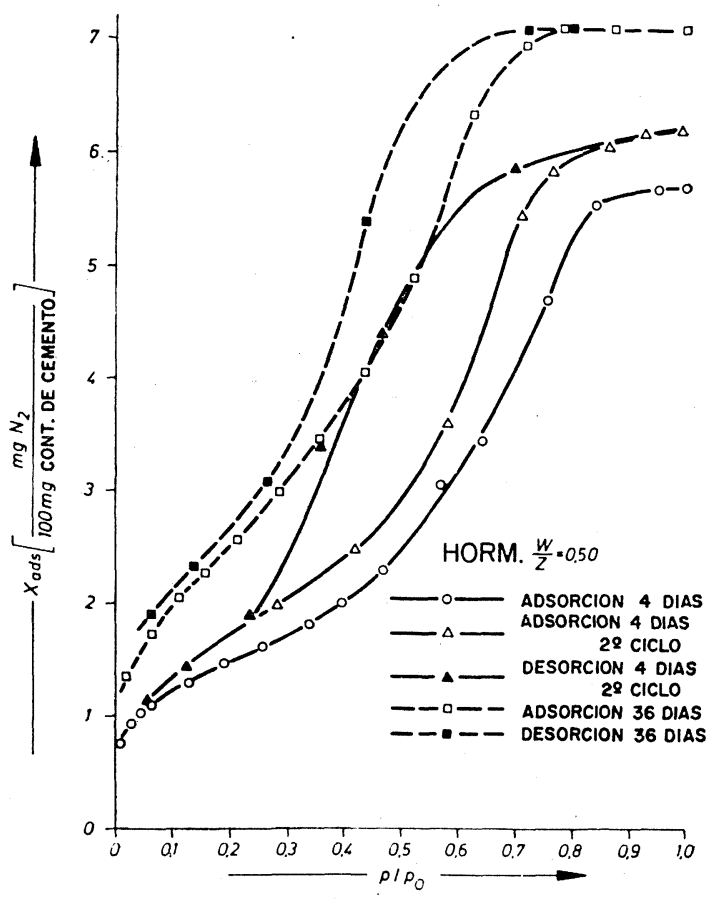

Fig. 11.-Medida gravimétrica de la sorción de $\mathbf{N}_{2}$; las isotermas de absorción $y$ desorción de diferentes edades de un hormipón $(a / c=0,50)$.

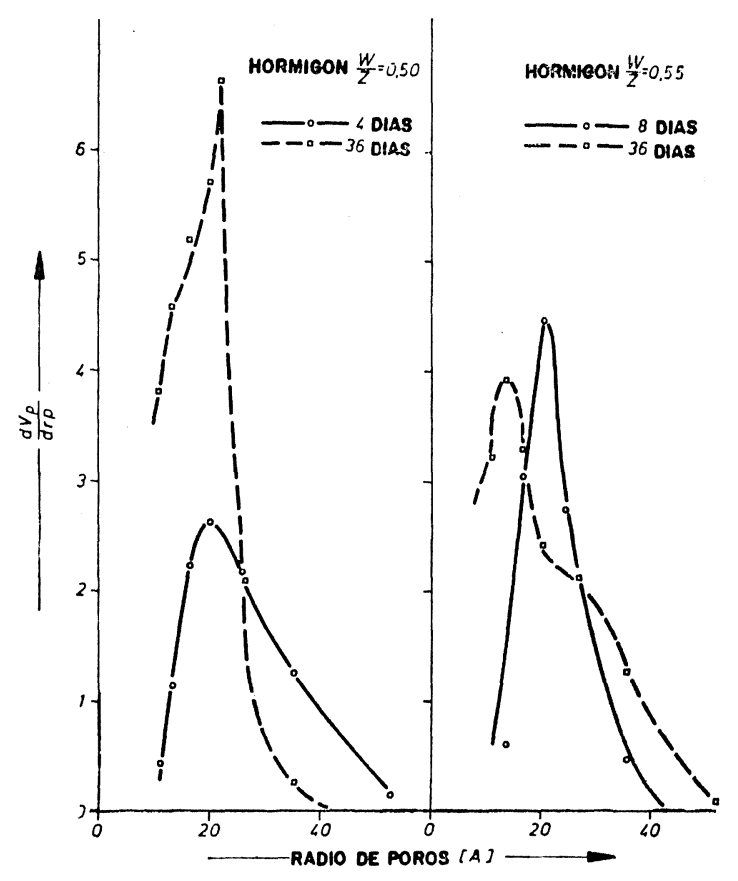

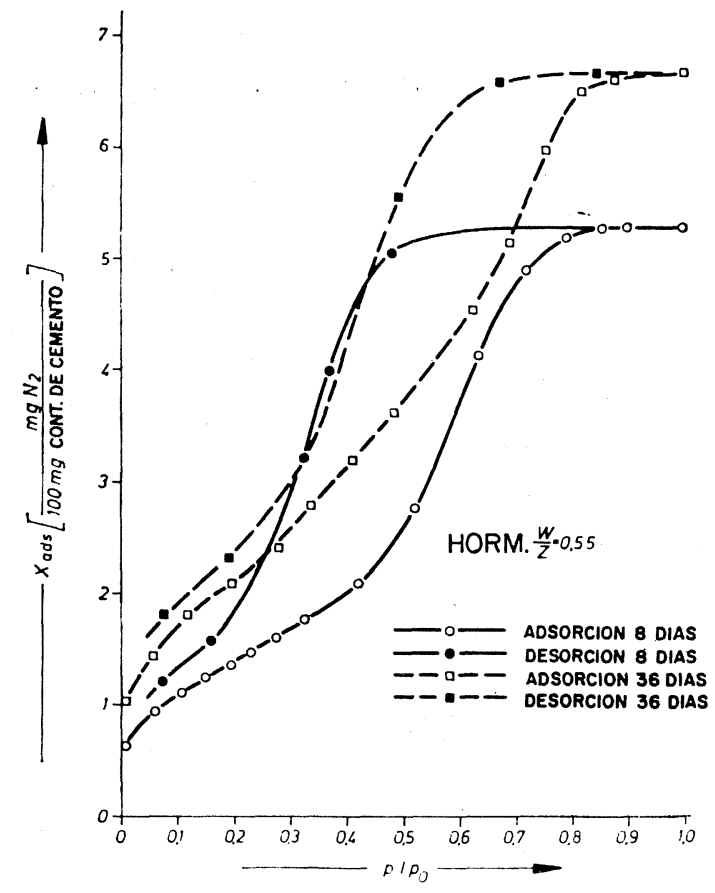

Fig. 12.-Medida gravimétrica de la sorción de $\mathbf{N}_{2}$; las isotermas de absorción y desorción de diferentes edades de un hormigón $(a / c=0,55)$.
Fig. 13.-Medida gravimétrica de la sorción de $\mathbf{N}_{2}$; comparación de la distribución de un valor de radios de los poros en hormigones de diferentes edades. $(a / c=0,50 \mathrm{y}$ 0,55 ) calculadas a partir de las isotermas de adsorción. 
No se puede conocer la dependencia existente entre el valor a/c y la distribución de la magnitud de poros ni siquiera en los análisis efectuados con ayuda del procedimiento de sorción de $\mathrm{N}_{2}$, lo cual hay que atribuir claramente a las reducidas diferencias de los valores a/c. Daimon, Akiba y. Kondo (14) efectuaron en tiempo muy reciente medidas por un procedimiento de permeabilidad en morteros de cemento con valores de a/c de 0,50 y 0,65 , consiguiendo resultados que presentan una buena coincidencia con los presentados aquí. Tal como se esperaba obtuvieron para el valor superior de a/c una gran porosidad de conjunto.

\section{DISCUSION DE LOS RESULTADOS}

Con el procedimiento de sorción de $\mathrm{N}_{2}$ se puede comprobar en el hormigón de diferente edad claras variaciones de las distribuciones de magnitud de poros. Con la progresiva hidratación o progreso de madurez de la pasta de cemento aumenta la parte de los poros pequeños, y concretamente a costa del espacio del conjunto de poros. Esto corresponde a las ideas que constituyen la base del modelo de estructura desarrollado por Powers (15). Los productos que se originan con la hidrólisis, hidratación y disposición penetran en los espacios de los poros primitivamente llenos de agua, lo cual provoca una mayor superficie del conjunto de poros con un aumento simultáneo (numérico) de poros muy pequeños (poros de gel).

No se puede comprobar la existencia de una dependencia entre el valor a/c y la distribución de la magnitud de los poros dentro de este análisis limitado. Sin embargo son de esperar diferencias en la porosidad de conjunto con valores de a/c que varían más intensamente entre sí.

Con el procedimiento del porosímetro de mercurio no pudieron captarse los máximos de las distribuciones de magnitud de poros, no obstante se pudieron conocer mediante estos análisis los desplazamientos característicos del progreso de madurez de las distribuciones de la magnitud de poros hacia poros más pequeños.

Con vistas a la caracterización de la estructura de los poros de hormigón hay que tender a una captación completa del espectro total de poros, pues su desarrollo es determinante para el progreso de madurez del hormigón. Por ello se presenta la aplicación del método del porosímetro de mercurio en unión con el procedimiento de absorción de $\mathrm{N}_{2}$, para poder determinar las distribuciones de poros de radios de 10 a $75 \times 10^{3} \AA$.

\section{RESUMEN}

En el mortero de cemento y en el hormigón se analizaron, con ayuda de la porosimetría de mercurio y del procedimiento de absorción de $\mathrm{N}_{2}$, las distribuciones de la magnitud de los poros. Los resultados muestran claras dependencias de las distribuciones y de la magnitud de poros respecto de la edad del material a ensayar. Las modificaciones de la estructura de los poros de la pasta de cemento pueden utilizarse para caracterizar el progreso de madurez del hormigón.

\section{B I B L I O G R A F I A}

(1) BRUnauer, S.: The structure of hardened Portland cement paste and concrete. Proceedings of the Eighth Conference on the Silicate Industry, Budapest (1965), S. 205-230.

(2) Schwiete, H. E., Ludwig, U.: Utber die Bestimmung der offenen Porosität in Zementstein. Tonind.-Ztg. 90 (1966), S. 562-574. 
(3) Astbury, N. F., Vyse, J.: A new method for the study of pore size distribution. Trans. Brit. Ceram. Soc. 10 (1971), S. $77-85$.

(4) RitteR, H. L., Drake, L. C.: Pore size distribution in porous materials. Ind. Engng. Chem. 17 (1945), S. $782-786$.

(5) Cranston, R. W., Inkley, F. A.: The determination of pore structures from nitrogen adsorption isotherms. Advances in Catalysis 9 (1957), S. 143-154.

(6) ORR, C., Dalla Valle. J. M.: Fine particle measurement. Size, surface and pore volume. New York: MacMillan 1959, S. 307-349.

(7) GuYer, A. Jr., Bohlen. B., Guyer, A.: Über die Bestimmung von Porengrößen. Helv. chim. acta 17 (1959). S. 2.103-2.110.

(8) Copeland, L. E., Hayes, J. C.: Determination of nonevaporable water in hardened Portland cement paste. ASTM Bull. 194 (1953), S. 70-74.

(9) Kroone, B., Blakey, F. A.: Reaction between carbon dioxide gas and mortar. J. Amer. Concr. Inst. 31 (1959), S. 497-510.

(10) Brunauer, S., Emmetr, P. H., Teller, E.: Adsorption of gases in ultimolecular layers. J. Amer. Chem. Soc. 60 (1938), S. 309 .

(11) Brunauer, S., Deming, L. S., Deming, W. S., Teller, E.: J. Amer. Chem. Soc. 62 (1940), S. 1.723.

(12) Mikhail. R. S., Copeland, L. E., Brunauer, S.: Pore structures and surface areas of hardened Portland cement pastes by nitrogen adsorption. Canad. J. Chem. 42 (1964), S. 426-438.

(13) LIPpens, B. C., DE BoeR, J. H.: Studies on pore systems in catalysts. J. of Catal. 4 (1965), S. 319-323.

(14) DaImon, H., AkIBA, T., Kondo. R.: Through pore size distribution and kinetics of the carbonation reaction of Portland cement mortars. J. Amer. Ceram. Soc. 54 (1971), S. 423-428.

(15) Powers, T. C.: Physical properties of cement paste. Proceedings of the 1th International Symposium on Chemistry of Cement, Washington (1960).

(16) GregG, S. J., Sing, K. S. W.: Adsorption, surface, area and porosity. London and New York: Academic Press 1967, second Printing 1969, S. 162-165. 